\title{
Curriculum Vitae: Alyssa A. Goodman
}

February 15, 2022

$\begin{array}{ll}\begin{array}{l}\text { Name } \\ \text { Office }\end{array} & \begin{array}{l}\text { Alyssa A. Goodman } \\ \text { Astronomy Department, Harvard University } \\ \text { Cambridge, MA 02138, 617-495-9278 }\end{array} \\ \text { Home } & \text { 485 Concord Avenue, Lexington, MA } 02421 \\ \text { Web Site } & \begin{array}{l}\text { scholar.harvard.edu/agoodman } \\ \text { Origin }\end{array} \\ \text { July 1, 1962, New York, New York }\end{array}$

\section{Education}

1984 Sc.B. Sc.B. Massachusetts Institute of Technology, Physics

Thesis: Grain Alignment in Molecular Clouds (Advisor: C. Alcock)

1986 A.M. Harvard University, Physics

1989 Ph.D. Harvard University, Physics

Thesis: Interstellar Magnetic Fields: An Observational Perspective (Advisor: P. Myers)

\section{Academic Experience}

$\begin{array}{ll}2015- & \text { Robert Wheeler Willson Professor of Applied Astronomy, Harvard University } \\ \text { 1995- } & \text { Research Associate, Smithsonian Astrophysical Observatory } \\ 2017-2021 & \text { Co-Director for Science, Radcliffe Insitute for Advanced Study } \\ 2017 & \text { Visiting Professor, University of Vienna } \\ 2016-2017 & \text { Edward, Frances, and Shirley B. Daniels Fellow, Radcliffe Institute for Advanced Study } \\ 2009-2010 & \text { Scholar-in-Residence, WGBH Boston (consultant role) } \\ 2008-2009 & \text { Scholar-in-Residence, WGBH Boston (sabbatical) } \\ 2005-2008 & \text { Founding Director, Harvard Initiative in Innovative Computing } \\ 2002-2010 & \text { Principal Investigator, The COMPLETE Survey of Star-Forming Regions } \\ 2001-2002 & \text { Visiting Professor, Yale University Astronomy Department (sabbatical) } \\ 1999-2015 & \text { Professor, Harvard University Astronomy Department } \\ 1996-1999 & \text { Associate Professor, Harvard University Astronomy Department } \\ 1995-1997 & \text { Head Tutor, Harvard University Astronomy Department } \\ 1992-1996 & \text { Assistant Professor, Harvard University Astronomy Department } \\ 1989-1992 & \text { Post-doctoral Fellow, University of California, Berkeley } \\ 1984-1989 & \text { Research Assistant, Harvard-Smithsonian Center for Astrophysics } \\ 1983 & \text { Summer Fellow, NASA-Goddard Institute for Space Studies }\end{array}$

\section{Other Professional Experience}

2020- Founder and President, glue solutions inc. 


\title{
Honors, Awards and Elected Positions
}

\author{
2020 \\ 2020 \\ 2019 \\ 2017 \\ 2015 \\ 2014 \\ 2014 \\ 2013 \\ 2009 \\ 2009 \\ 2008 \\ 2008- \\ 2008 \\ 2008 \\ 2006 \\ 2005 \\ 2004 \\ 1998 \\ 1997 \\ 1994-2000 \\ 1994 \\ 1993-1995 \\ 1989-1991 \\ 1990 \\ 1986-1989 \\ 1985 \\ 1983 \\ Franke Drake Lectureship (delivered 2022), Green Bank Observatory \\ Fellow, American Astronomical Society (AAS) \\ Tinsley Lecturer, University of Texas \\ Salpeter Lecturer, Cornell University \\ Scientist of the Year, Harvard Foundation \\ Pappalardo Lecturer, Massachusetts Institute of Technology \\ Benjamin Dean Lecturer, California Academy of Sciences \\ Bishop Lecturer, Columbia University \\ Fellow, American Association for the Advancement of Science (AAAS) \\ Collins Lecturer, Massachusetts General Hospital \\ Inaugural "Scholar-in-Residence" at WGBH, Boston \\ Microsoft Academic Partner \\ Apple Science Innovator Award \\ Chair, Astronomy Section of the AAAS \\ NCSA Distinguished Lecturer, National Center for Supercomputer Applications \\ Dean's Distinguished Lecturer, Harvard School of Public Health \\ Sturm Lecturer, Wesleyan University \\ Bok Prize, Harvard University \\ Newton Lacy Pierce Prize, American Astronomical Society \\ National Science Foundation Young Investigator \\ Pedagogical Innovation Award, Harvard University \\ Alfred P. Sloan Fellow \\ President's Fellowship, University of California, Berkeley \\ First Prize Paper, NATO ASI on Star Formation \\ Amelia Earhart Fellowships from Zonta International \\ Francis Lee Freidman Award in Physics, Harvard University \\ Sigma Pi Sigma, MIT
}

\section{Society Memberships}

American Association for the Advancement of Science; American Association of University Professors; American Astronomical Society; International Astronomical Union; Association for Computing Machinery; IEEE; URSI Commission J (Radio Astronomy)

\section{Advisory \& Review Committees}

NASA-Infrared Space Observatory Key Projects Review (1992); Scientific Working Group for NRAO Green Bank Telescope (1992-99); Arecibo Users and Scientific Advisory Committee (1993-96); NSF Site Review for Center for Particle Astrophysics (1994); NASA Astrophysics Data Program Review (1995); Smithsonian Astrophysical Observatory Time Allocation Committee (1995-97); NSF-Caltech Submillimeter Observatory Review (1996); NSF Galactic Astronomy ISM Panel (1996, 2000 (chair)); M4 Satellite Science Advisory Group, Chair (1997, 2000); Harvard University Faculty Council (1997-98); AAS Publications Board (1998-2001); United States Square Kilometer Array Consortium Representative (1999-); National Academy of Science's Committee on Astronomy and Astrophysics (2000-2003); NASA-SIRTF Legacy Review, Panel Chair (2000); AAS Committee on Astronomy and Public Policy (2000-6); NRAO VLA/VLBA Time Allocation Committee (2001-4); AUI NRAO Director Search Committee (2002); Harvard University Provost's Committee on Science (2002-3); Task Force on Science and Technology (Harvard, 20034); Center for Astrophysics Director Search Advisory Committee (2003-4); Spitzer Science Center Oversight Committee (2003-6); Harvard Commission of Inquiry (2004-5, 2007-8); Harvard Subcommittee on Quantitative Reasoning (2002-2008); Goldwater Scholarship Selection Committee (2004-); Harvard FAS Dean Search Advisory Committee (2006-7); AAS Chambliss Award (for Textbook Writing) Committee (2006-7); Yale University Visiting Committee for Astronomy (2008); AAAS Chair (2008); AAAS Board (2009); NRC board on Research Data and Information (2009-2012); VAO Scientific Advisory Committee (2009-2012); ALMA North American Science Advisory Committee 
(2009-2012); Graduate Admissions, Chair (2011, 2014); Mentoring Committee, Chair (2011); Harvard Library Digital Scholarship Committee (2011-2012); Committee on Professional Conduct (2011-); HILT Advisory Committee (2012-2014); Faculty Advisor to the Communication Science Conference (ComSciCon, 2014-); Harvard/FAS Library Committee (2014-2019); American Astronomical Society Publications Task Force (2014); American Astronomical Society Publications WorldWide Telescope Force (2015); Astroinformatics and Astrostatistics Working Group of the American Astronomical Society (2012-); Sloan/UCLA Knowledge Infrastructures Advisory Committee (2012-); Board on Data, Ethics and Society (2014-2017); Dunlap Institute Advisory Committee (Chair, 2016-2019); Harvard Data Science Initiative Steering Committee (2017-2019); Vice Provost for Advances in Learning Advisory Committee (2017-19); Harvard FAS-Division of Continuing Education Standing Committee (Chair) (2019-); MPE Fachtbeirat (2019); CEASAR Advisory Board (2020-); (German) National Research Data Infrastructures (NFDI) Review Panel (2020); ORIGINS Excellence Cluster (Germany) Science Advisory Committee (2020-); NAS Committee on Astronomy and Astrophysics (2021-); NASA SciAct Working Group on The Science of Science (2021-) [Departmental and Conference Organizing Committees not listed here.]

\section{References}

2. Grasser N, Ratzenböck S, Alves J, Großschedl J, Meingast S, Zucker C, Hacar A, Lada C, Goodman A, Lombardi M, Forbes JC, Bomze IM, Möller T (2021) The $\rho$ Oph region revisited with Gaia EDR3. arXiv e-prints, :arXiv:2101.12200.

3. Swiggum C, D'Onghia E, Alves J, Großschedl J, Foley M, Zucker C, Meingast S, Chen B, Goodman A (2021) Evidence for Radial Expansion at the Core of the Orion Complex with Gaia EDR3. arXiv e-prints, :arXiv:2101.10380.

4. Schmiedeke A, Pineda JE, Caselli P, Arce HG, Fuller GA, Goodman AA, José Maureira M, Offner SSR, SeguraCox D, Seifried D (2021) Dissecting the super-critical filaments embedded in the 0.5 pc subsonic region of Barnard 5. arXiv e-prints, :arXiv:2101.00248.

5. Kong S, Ossenkopf-Okada V, Arce HG, Bally J, Sánchez-Monge Á, McGehee P, Suri S, Klessen RS, Carpenter JM, Lis DC, Nakamura F, Schilke P, Smith RJ, Mairs S, Goodman A, Maureira MJ (2021) The CARMA-NRO Orion Survey: Filament Formation via Collision-induced Magnetic Reconnection-the Stick in Orion A. ApJ, 906(2):80. https://doi.org/10.3847/1538-4357/abc687

6. Das KK, Zucker C, Speagle JS, Goodman A, Green GM, Alves J (2020) Constraining the distance to the North Polar Spur with Gaia DR2. MNRAS, 498(4):5863-5872. https://doi.org/10.1093/mnras/staa2702

7. Sridharan TK, Bialy S, Blundell R, Burkhardt A, Dame T, Doeleman S, Finkbeiner D, Goodman A, Grimes P, Imara N, Johnson M, Keating G, Lada C, Le Gal R, Myers P, Narayan R, Paine S, Patel N, Raymond A, Tong E, Wilner D, Zhang Q, Zucker C (2020) A Prospective ISRO-CfA Himalayan Sub-millimeter-wave Observatory Initiative. arXiv e-prints, :arXiv:2008.07453.

8. Choudhury S, Pineda JE, Caselli P, Ginsburg A, Offner SSR, Rosolowsky E, Friesen RK, Alves FO, ChacónTanarro A, Punanova A, Redaelli E, Kirk H, Myers PC, Martin PG, Shirley Y, Chun-Yuan Chen M, Goodman AA, Di Francesco J (2020) Ubiquitous $\mathrm{NH}_{3}$ supersonic component in L1688 coherent cores. A\&SA, 640:L6. https: //doi.org/10.1051/0004-6361/202037955

9. Chen HH-H, Offner SSR, Pineda JE, Goodman AA, Burkert A, Ginsburg A, Choudhury S (2020) Core Formation, Coherence and Collapse: A New Core Evolution Paradigm Revealed by Machine Learning. arXiv e-prints, :arXiv:2006.07325.

10. Harju J, Pineda JE, Vasyunin AI, Caselli P, Offner SSR, Goodman AA, Juvela M, Sipilä O, Faure A, Le Gal R, Hily-Blant P, Alves J, Bizzocchi L, Burkert A, Chen H, Friesen RK, Güsten R, Myers PC, Punanova A, Rist C, Rosolowsky E, Schlemmer S, Shirley Y, Spezzano S, Vastel C, Wiesenfeld L (2020) Efficient Methanol Production on the Dark Side of a Prestellar Core. ApJ, 895(2):101. https://doi.org/10.3847/1538-4357/ab8f93

11. Chen C-Y, Behrens EA, Washington JE, Fissel LM, Friesen RK, Li Z-Y, Pineda JE, Ginsburg A, Kirk H, Scibelli S, Alves F, Redaelli E, Caselli P, Punanova A, Di Francesco J, Rosolowsky E, Offner SSR, Martin PG, ChacónTanarro A, Chen HH-H, Chen MC-Y, Keown J, Seo Y, Shirley Y, Arce HG, Goodman AA, Matzner CD, Myers PC, Singh A (2020) Relative alignment between dense molecular cores and ambient magnetic field: the synergy of numerical models and observations. MNRAS, 494(2):1971-1987. https://doi.org/10.1093/mnras/staa835 
12. Alves J, Zucker C, Goodman AA, Speagle JS, Meingast S, Robitaille T, Finkbeiner DP, Schlafly EF, Green GM (2020) A Galactic-scale gas wave in the solar neighbourhood. Nature, 578(7794):237-239. https://doi.org/10. $1038 / \mathrm{s} 41586-019-1874-z$

13. Zucker C, Speagle JS, Schlafly EF, Green GM, Finkbeiner DP, Goodman A, Alves J (2020) A compendium of distances to molecular clouds in the Star Formation Handbook. A\&SA,633:A51. https://doi.org/10.1051/0004$6361 / 201936145$

14. Stephens IW, Bourke TL, Dunham MM, Myers PC, Pokhrel R, Tobin JJ, Arce HG, Sadavoy SI, Vorobyov EI, Pineda JE, Offner SSR, Lee KI, Kristensen LE, Jørgensen JK, Gurwell MA, Goodman AA (2019) Mass Assembly of Stellar Systems and Their Evolution with the SMA (MASSES) - Full Data Release. ApJS, 245(2):21. https: //doi.org/10.3847/1538-4365/ab5181

15. Zucker C, Smith R, Goodman A (2019) Synthetic Large-scale Galactic Filaments: On Their Formation, Physical Properties, and Resemblance to Observations. ApJ, 887(2):186. https://doi.org/10.3847/1538-4357/ab517d

16. Chen HH-H, Pineda JE, Offner SSR, Goodman AA, Burkert A, Friesen RK, Rosolowsky E, Scibelli S, Shirley Y (2019) Droplets. II. Internal Velocity Structures and Potential Rotational Motions in Pressure-dominated Coherent Structures. ApJ, 886(2):119. https://doi.org/10.3847/1538-4357/ab4ce9

17. Houghton H, Udomprasert P, Sunbury S, Wright E, Goodman A, Johnson E, Bishop A (2019) Cultivating Curiosity with Life in the Universe and WorldWide Telescope. Advancing Astronomy for All: ASP 2018, 524:273.

18. Udomprasert P, Houghton H, Sunbury S, Plummer J, Wright E, Goodman A, Johnson E, Zhang H, Vaishampayan A, Cho K (2019) Visualizing Seasons and Moon Phases with WorldWide Telescope. Advancing Astronomy for All: ASP 2018, 524:125.

19. Zucker C, Speagle JS, Schlafly E, Green GM, Finkbeiner DP, Goodman A, Alves J (2019) VizieR Online Data Catalog: Distances to molecular clouds in SFR (Zucker+, 2020). VizieR Online Data Catalog, :J/A+A/633/A51.

20. Zucker C, Speagle JS, Schlafly EF, Green GM, Finkbeiner DP, Goodman AA, Alves J (2019) A Large Catalog of Accurate Distances to Local Molecular Clouds: The Gaia DR2 Edition. ApJ, 879(2):125. https://doi.org/10. $3847 / 1538-4357 / \mathrm{ab} 2388$

21. Chen HH-H, Pineda JE, Goodman AA, Burkert A, Offner SSR, Friesen RK, Myers PC, Alves F, Arce HG, Caselli P, Chacón-Tanarro A, Chen MC-Y, Di Francesco J, Ginsburg A, Keown J, Kirk H, Martin PG, Matzner C, Punanova A, Redaelli E, Rosolowsky E, Scibelli S, Seo Y, Shirley Y, Singh A, The GAS Collaboration (2019) Droplets. I. Pressure-dominated Coherent Structures in L1688 and B18. ApJ, 877(2):93. https://doi.org/10.3847/1538$4357 / \mathrm{ab} 1 \mathrm{a} 40$

22. Siemiginowska A, Eadie G, Czekala I, Feigelson E, Ford EB, Kashyap V, Kuhn M, Loredo T, Ntampaka M, Stevens A, Avelino A, Borne K, Budavari T, Burkhart B, Cisewski-Kehe J, Civano F, Chilingarian I, van Dyk DA, Fabbiano G, Finkbeiner DP, Foreman-Mackey D, Freeman P, Fruscione A, Goodman AA, Graham M, Guenther HM, Hakkila J, Hernquist L, Huppenkothen D, James DJ, Law C, Lazio J, Lee T, López-Morales M, Mahabal AA, Mandel K, Meng X-L, Moustakas J, Muna D, Peek JEG, Richards G, Portillo SKN, Scargle J, de Souza RS, Speagle JS, Stassun KG, Stenning DC, Taylor SR, Tremblay GR, Trimble V, Yanamand ra-Fisher PA, Young CA (2019) The Next Decade of Astroinformatics and Astrostatistics. BAAS, 51(3):355.

23. Ntampaka M, Avestruz C, Boada S, Caldeira J, Cisewski-Kehe J, Di Stefano R, Dvorkin C, Evrard AE, Farahi A, Finkbeiner D, Genel S, Goodman A, Goulding A, Ho S, Kosowsky A, La Plante P, Lanusse F, Lochner M, Mandelbaum R, Nagai D, Newman JA, Nord B, Peek JEG, Peel A, Poczos B, Rau MM, Siemiginowska A, Sutherland DJ, Trac H, Wandelt B (2019) The Role of Machine Learning in the Next Decade of Cosmology. BAAS, 51(3):14.

24. Soler JD, Beuther H, Rugel M, Wang Y, Clark PC, Glover SCO, Goldsmith PF, Heyer M, Anderson LD, Goodman A, Henning T, Kainulainen J, Klessen RS, Longmore SN, McClure-Griffiths NM, Menten KM, Mottram JC, Ott J, Ragan SE, Smith RJ, Urquhart JS, Bigiel F, Hennebelle P, Roy N, Schilke P (2019) Histogram of oriented gradients: a technique for the study of molecular cloud formation. A\&A,622:A166. https://doi.org/10.1051/ 0004-6361/201834300

25. Zucker C, Schlafly EF, Speagle JS, Green GM, Portillo SKN, Finkbeiner DP, Goodman AA (2018) Mapping Distances across the Perseus Molecular Cloud Using CO Observations, Stellar Photometry, and Gaia DR2 Parallax Measurements. ApJ, 869(1):83. https://doi.org/10.3847/1538-4357/aae97c 
26. Grossschedl JE, Alves J, Meingast S, Ackerl C, Ascenso J, Bouy H, Burkert A, Forbrich J, Fuernkranz V, Goodman A, Hacar A, Herbst-Kiss G, Lada CJ, Larreina I, Leschinski K, Lombardi M, Moitinho A, Mortimer D, Zari E (2018) VizieR Online Data Catalog: 3D shape of Orion A from Gaia DR2 (Grossschedl+, 2018). VizieR Online Data Catalog, :J/A+A/619/A106.

27. Großschedl JE, Alves J, Meingast S, Ackerl C, Ascenso J, Bouy H, Burkert A, Forbrich J, Fürnkranz V, Goodman A, Hacar Á, Herbst-Kiss G, Lada CJ, Larreina I, Leschinski K, Lombardi M, Moitinho A, Mortimer D, Zari E (2018) 3D shape of Orion A from Gaia DR2. A\&A, 619:A106. https://doi.org/10.1051/0004-6361/201833901

28. Zucker C, Battersby C, Goodman A (2018) Physical Properties of Large-scale Galactic Filaments. ApJ, 864(2):153. https://doi.org/10.3847/1538-4357/aacc66

29. Stephens IW, Dunham MM, Myers PC, Pokhrel R, Bourke TL, Vorobyov EI, Tobin JJ, Sadavoy SI, Pineda JE, Offner SSR, Lee KI, Kristensen LE, Jørgensen JK, Goodman AA, Arce HG, Gurwell M (2018) Mass Assembly of Stellar Systems and Their Evolution with the SMA (MASSES) - 1.3 mm Subcompact Data Release. ApJS, $237(2): 22$. https://doi.org/10.3847/1538-4365/aacda9

30. Allen G, Anderson W, Blaufuss E, Bloom JS, Brady P, Burke-Spolaor S, Cenko SB, Connolly A, Couvares P, Fox D, Gal-Yam A, Gezari S, Goodman A, Grant D, Groot P, Guillochon J, Hanna C, Hogg DW, Holley-Bockelmann K, Howell DA, Kaplan D, Katsavounidis E, Kowalski M, Lehner L, Muthukrishna D, Narayan G, Peek JEG, Saha A, Shawhan P, Taboada I (2018) Multi-Messenger Astrophysics: Harnessing the Data Revolution. arXiv e-prints, :arXiv:1807.04780.

31. Monsch K, Pineda JE, Liu HB, Zucker C, How-Huan Chen H, Pattle K, Offner SSR, Di Francesco J, Ginsburg A, Ercolano B, Arce HG, Friesen R, Kirk H, Caselli P, Goodman AA (2018) Dense Gas Kinematics and a Narrow Filament in the Orion A OMC1 Region Using $\mathrm{NH}_{3}$. ApJ, 861(2):77. https://doi .org/10.3847/1538-4357/aac8da

32. Kong S, Arce HG, Feddersen JR, Carpenter JM, Nakamura F, Shimajiri Y, Isella A, Ossenkopf-Okada V, Sargent AI, Sánchez-Monge Á, Suri ST, Kauffmann J, Pillai T, Pineda JE, Koda J, Bally J, Lis DC, Padoan P, Klessen R, Mairs S, Goodman A, Goldsmith P, McGehee P, Schilke P, Teuben PJ, Maureira MJ, Hara C, Ginsburg A, Burkhart B, Smith RJ, Schmiedeke A, Pineda JL, Ishii S, Sasaki K, Kawabe R, Urasawa Y, Oyamada S, Tanabe Y (2018) The CARMA-NRO Orion Survey. ApJS, 236(2):25. https://doi.org/10.3847/1538-4365/aabafc

33. Chen HH-H, Burkhart B, Goodman A, Collins DC (2018) The Anatomy of the Column Density Probability Distribution Function (N-PDF). ApJ, 859(2):162. https://doi.org/10.3847/1538-4357/aabaf6

34. Kirk H, Friesen RK, Pineda JE, Rosolowsky E, Offner SSR, Matzner CD, Myers PC, di FJ, Caselli P, Alves FO, Chacon-Tanarro A, Chen H-H, Chen MC-Y, Keown J, Punanova A, Seo YM, Shirley Y, Ginsburg A, Hall C, Singh A, Arce HG, Goodman AA, Martin P, Redaelli E (2018) VizieR Online Data Catalog: Virial analysis of the dense cores in Orion A (Kirk+, 2017). VizieR Online Data Catalog, :J/ApJ/846/144.

35. Goodman AA, Borkin MA, Robitaille TP (2018) New Thinking on, and with, Data Visualization. arXiv e-prints, :arXiv:1805.11300.

36. Rosenfield P, Fay J, Gilchrist RK, Cui C, Weigel AD, Robitaille T, Otor OJ, Goodman A (2018) AAS WorldWide Telescope: A Seamless, Cross-platform Data Visualization Engine for Astronomy Research, Education, and Democratizing Data. ApJS, 236(1):22. https://doi.org/10.3847/1538-4365/aab776

37. Keown J, Di Francesco J, Kirk H, Friesen RK, Pineda JE, Rosolowsky E, Ginsburg A, Offner SSR, Caselli P, Alves F, Chacón-Tanarro A, Punanova A, Redaelli E, Seo YM, Matzner CD, Chun-Yuan Chen M, Goodman AA, Chen H-H, Shirley Y, Singh A, Arce HG, Martin P, Myers PC (2017) The Green Bank Ammonia Survey: Observations of Hierarchical Dense Gas Structures in Cepheus-L1251. ApJ, 850(1):3. https://doi.org/10.3847/1538-4357/ aa93ec

38. Kirk H, Friesen RK, Pineda JE, Rosolowsky E, Offner SSR, Matzner CD, Myers PC, Di Francesco J, Caselli P, Alves FO, Chacón-Tanarro A, Chen H-H, Chun-Yuan Chen M, Keown J, Punanova A, Seo YM, Shirley Y, Ginsburg A, Hall C, Singh A, Arce HG, Goodman AA, Martin P, Redaelli E (2017) The Green Bank Ammonia Survey: Dense Cores under Pressure in Orion A. ApJ, 846(2):144. https://doi.org/10.3847/1538-4357/aa8631

39. Stephens IW, Dunham MM, Myers PC, Pokhrel R, Sadavoy SI, Vorobyov EI, Tobin JJ, Pineda JE, Offner SSR, Lee KI, Kristensen LE, Jørgensen JK, Goodman AA, Bourke TL, Arce HG, Plunkett AL (2017) Alignment between Protostellar Outflows and Filamentary Structure. ApJ, 846(1):16. https://doi.org/10.3847/1538-4357/aa8262 
40. Anderson LD, Wang Y, Bihr S, Rugel M, Beuther H, Bigiel F, Churchwell E, Glover SCO, Goodman AA, Henning T, Heyer M, Klessen RS, Linz H, Longmore SN, Menten KM, Ott J, Roy N, Soler JD, Stil JM, Urquhart JS (2017) Galactic supernova remnant candidates discovered by THOR. A\&A, 605:A58. https://doi.org/10.1051/0004$6361 / 201731019$

41. Friesen RK, Pineda JE, co-PIs, Rosolowsky E, Alves F, Chacón-Tanarro A, How-Huan Chen H, Chun-Yuan Chen M, Di Francesco J, Keown J, Kirk H, Punanova A, Seo Y, Shirley Y, Ginsburg A, Hall C, Offner SSR, Singh A, Arce HG, Caselli P, Goodman AA, Martin PG, Matzner C, Myers PC, Redaelli E, GAS Collaboration (2017) The Green Bank Ammonia Survey: First Results of $\mathrm{NH}_{3}$ Mapping of the Gould Belt. ApJ, 843(1):63. https://doi.org/10.3847/1538-4357/aa6d58

42. Hull CLH, Mocz P, Burkhart B, Goodman AA, Girart JM, C. CP, Hernquist L, Springel V, Zhi-Yun L, Lai S-P (2017) Unveiling the Role of the Magnetic Field at the Smallest Scales of Star Formation. ApJ, 842:L9. https: //doi.org/10.3847/2041-8213/aa71b7

43. Zook M, Barocas S, boyd danah, Kate C, Keller E, Gangadharan SP, Goodman A, Hollander R, A. KB, Metcalf J, Narayanan A, Nelson A, Pasquale F (2017) Ten simple rules for responsible big data research. PLoS Computational Biology, 13:e1005399. https://doi.org/10.1371/journal.pcbi.1005399

44. Pillitteri I, Wolk SJ, Chen HH, Goodman A (2016) First stars of the $\rho$ Ophiuchi dark cloud. XMM-Newton view of $\rho$ Oph and its neighbors. A\&SA, 592:A88. https://doi.org/10.1051/0004-6361/201628284

45. Rice TS, Goodman AA, Bergin EA, Beaumont C, Dame TM (2016) A Uniform Catalog of Molecular Clouds in the Milky Way. ApJ, 822:52. https://doi.org/10.3847/0004-637X/822/1/52

46. Lee KI, Dunham MM, Myers PC, Arce HG, Bourke TL, Goodman AA, Jørgensen JK, Kristensen LE, Offner SSR, Pineda JE, Tobin JJ, Vorobyov EI (2016) Misalignment of Outflow Axes in the Proto-multiple Systems in Perseus. ApJL, 820:L2. https://doi.org/10.3847/2041-8205/820/1/L2

47. Meingast S, Alves J, Mardones D, Teixeira PS, Lombardi M, Großschedl J, Ascenso J, Bouy H, Forbrich J, Goodman A, Hacar A, Hasenberger B, Kainulainen J, Kubiak K, Lada C, Lada E, Moitinho A, Petr-Gotzens M, Rodrigues L, Román-Zúñiga CG (2016) VISION - Vienna survey in Orion. I. VISTA Orion A Survey. AEAA, 587:A153. https://doi.org/10.1051/0004-6361/201527160

48. Lee KI, Dunham MM, Myers PC, Tobin JJ, Kristensen LE, Pineda JE, Vorobyov EI, Offner SSR, Arce HG, Li Z-Y, Bourke TL, Jørgensen JK, Goodman AA, Sadavoy SI, Chandler CJ, Harris RJ, Kratter K, Looney LW, Melis C, Perez LM, Segura-Cox D (2015) Mass Assembly of Stellar Systems and Their Evolution with the SMA (MASSES). Multiplicity and the Physical Environment in L1448N. ApJ, 814:114. https://doi.org/10.1088/0004$637 \mathrm{X} / 814 / 2 / 114$

49. Zucker C, Battersby C, Goodman A (2015) The Skeleton of the Milky Way. ApJ, 815:23. https://doi.org/ $10.1088 / 0004-637 X / 815 / 1 / 23$

50. Ting Y-S, Conroy C, Goodman A (2015) Prospects for Chemically Tagging Stars in the Galaxy. ApJ, 807:104. https://doi.org/10.1088/0004-637X/807/1/104

51. Pineda JE, Offner SSR, Parker RJ, Arce HG, Goodman AA, Caselli P, Fuller GA, Bourke TL, Corder SA (2015) The formation of a quadruple star system with wide separation. Nature, 518:213-215. https://doi.org/10.1038/ nature14166

52. Goodman AA, Alves J, Beaumont CN, Benjamin RA, Borkin MA, Burkert A, Dame TM, Jackson J, Kauffmann J, Robitaille T, Smith RJ (2014) The Bones of the Milky Way. ApJ, 797:53. https://doi.org/10.1088/0004$637 X / 797 / 1 / 53$

53. Beaumont CN, Goodman AA, Kendrew S, Williams JP, Simpson R (2014) The Milky Way Project: Leveraging Citizen Science and Machine Learning to Detect Interstellar Bubbles. ApJS, 214:3. https://doi.org/10.1088/ 0067-0049/214/1/3

54. Pepe A, Goodman A, Muench A, Crosas M, Erdmann C (2014) How Do Astronomers Share Data? Reliability and Persistence of Datasets Linked in AAS Publications and a Qualitative Study of Data Practices among US Astronomers. PLoS ONE, 9(10):104798. https://doi.org/10.1371/journal.pone.0104798

55. Pillitteri I, Wolk SJ, Goodman A, Sciortino S (2014) Smooth X-ray variability from $\rho$ Ophiuchi A+B. A strongly magnetized primary B2 star?. AESA, 567:L4. https://doi.org/10.1051/0004-6361/201424243 
56. Sanders NE, Faesi C, Goodman AA (2014) A New Approach to Developing Interactive Software Modules Through Graduate Education. Journal of Science Education and Technology, 23:431-440. https://doi.org/10. $1007 / \mathrm{s} 10956-013-9474-4$

57. Goodman A, Pepe A, Blocker AW, Borgman CL, Cranmer K, Crosas M, Di Stefano R, Gil Y, Groth P, Hedstrom M, Hogg DW, Kashyap V, Mahabal A, Siemiginowska A, Slavkovic A (2014) Ten Simple Rules for the Care and Feeding of Scientific Data. PLoS Computational Biology, 10:3542. https://doi.org/10.1371/journal. pcbi. 1003542

58. Li H-B, Goodman A, Sridharan TK, Houde M, Li Z-Y, Novak G, Tang KS (2014) The Link Between Magnetic Fields and Cloud/Star Formation. Protostars and Planets VI, :101-123. https://doi.org/10.2458/azu_uapress_ 9780816531240-ch005

59. Beaumont CN, Offner SSR, Shetty R, Glover SCO, Goodman AA (2013) Quantifying Observational Projection Effects Using Molecular Cloud Simulations. ApJ, 777:173. https://doi.org/10.1088/0004-637X/777/2/173

60. Burkhart B, Lazarian A, Goodman A, Rosolowsky E (2013) Hierarchical Structure of Magnetohydrodynamic Turbulence in Position-position-velocity Space. ApJ, 770:141. https://doi.org/10.1088/0004-637X/770/2/141

61. Foster JB, Mandel KS, Pineda JE, Covey KR, Arce HG, Goodman AA (2013) Evidence for grain growth in molecular clouds: A Bayesian examination of the extinction law in Perseus. MNRAS, 428:1606-1622. https: //doi.org/10.1093/mnras/sts144

62. Beaumont CN, Goodman AA, Alves JF, Lombardi M, Román-Zúñiga CG, Kauffmann J, Lada CJ (2012) A simple perspective on the mass-area relationship in molecular clouds. MNRAS, 423:2579-2586. https://doi.org/ $10.1111 / \mathrm{j} .1365-2966.2012 .21061 . \mathrm{x}$

63. Kelly BC, Shetty R, Stutz AM, Kauffmann J, Goodman AA, Launhardt R (2012) Dust Spectral Energy Distributions in the Era of Herschel and Planck: A Hierarchical Bayesian-fitting Technique. ApJ, 752:55. https://doi.org/10.1088/0004-637X/752/1/55

64. Goodman AA (2012) Principles of high-dimensional data visualization in astronomy. Astronomische Nachrichten, 333:505. https://doi.org/10.1002/asna.201211705

65. Offner SSR, Capodilupo J, Schnee S, Goodman AA (2012) Observing turbulent fragmentation in simulations: predictions for CARMA and ALMA. MNRAS, 420:L53-L57. https://doi.org/10.1111/j.1745-3933.2011.01194.x

66. Arce HG, Borkin MA, Goodman AA, Pineda JE, Beaumont CN (2011) A Bubbling Nearby Molecular Cloud: COMPLETE Shells in Perseus. ApJ, 742:105. https://doi.org/10.1088/0004-637X/742/2/105

67. Pineda JE, Arce HG, Schnee S, Goodman AA, Bourke T, Foster JB, Robitaille T, Tanner J, Kauffmann J, Tafalla M, Caselli P, Anglada G (2011) The Enigmatic Core L1451-mm: A First Hydrostatic Core? Or a Hidden VeLLO?. ApJ, 743:201. https://doi.org/10.1088/0004-637X/743/2/201

68. Offner SSR, Lee EJ, Goodman AA, Arce H (2011) Radiation-hydrodynamic Simulations of Protostellar Outflows: Synthetic Observations and Data Comparisons. ApJ, 743:91. https://doi.org/10.1088/0004-637X/743/1/91

69. Beaumont CN, Williams JP, Goodman AA (2011) Classifying Structures in the Interstellar Medium with Support Vector Machines: The G16.05-0.57 Supernova Remnant. ApJ, 741:14. https://doi .org/10.1088/0004-637X/741/ $1 / 14$

70. Pineda JE, Goodman AA, Arce HG, Caselli P, Longmore S, Corder S (2011) Expanded Very Large Array Observations of the Barnard 5 Star-forming Core: Embedded Filaments Revealed. ApJL, 739:L2. https://doi. org/10.1088/2041-8205/739/1/L2

71. Goodman A, Joyce R, Smith JP (2011) The long shadow cast by childhood physical and mental problems on adult life. Proceedings of the National Academy of Science, 108:6032-6037. https://doi.org/10.1073/pnas.1016970108

72. Goodman AA (2011) A Guide to Comparisons of Star Formation Simulations with Observations. Computational Star Formation, 270:511-519. https://doi.org/10.1017/S1743921311000901

73. Schmalzl M, Kainulainen J, Quanz SP, Alves J, Goodman AA, Henning T, Launhardt R, Pineda JE, RománZúñiga CG (2010) Star Formation in the Taurus Filament L 1495: From Dense Cores to Stars. ApJ, 725:1327-1336. https://doi.org/10.1088/0004-637X/725/1/1327 
74. Dib S, Hennebelle P, Pineda JE, Csengeri T, Bontemps S, Audit E, Goodman AA (2010) The Angular Momentum of Magnetized Molecular Cloud Cores: A Two-dimensional-Three-dimensional Comparison. ApJ, 723:425-439. https://doi.org/10.1088/0004-637X/723/1/425

75. Kirk H, Pineda JE, Johnstone D, Goodman A (2010) The Dynamics of Dense Cores in the Perseus Molecular Cloud. II. The Relationship Between Dense Cores and the Cloud. ApJ, 723:457-475. https://doi.org/10.1088/ 0004-637X/723/1/457

76. Loinard L, Rodríguez LF, Gómez L, Cantó J, Raga AC, Goodman AA, Arce HG (2010) A reassessment of the kinematics of PV Cephei based on accurate proper motion measurements. RevMexAA, 46:375-383.

77. Arce HG, Borkin MA, Goodman AA, Pineda JE, Halle MW (2010) The COMPLETE Survey of Outflows in Perseus. ApJ, 715:1170-1190. https://doi.org/10.1088/0004-637X/715/2/1170

78. Kauffmann J, Pillai T, Shetty R, Myers PC, Goodman AA (2010) The Mass-size Relation from Clouds to Cores. II. Solar Neighborhood Clouds. ApJ, 716:433-445. https://doi.org/10.1088/0004-637X/716/1/433

79. Shetty R, Collins DC, Kauffmann J, Goodman AA, Rosolowsky EW, Norman ML (2010) The Effect of Projection on Derived Mass-Size and Linewidth-Size Relationships. ApJ, 712:1049-1056. https://doi.org/10.1088/0004$637 \mathrm{X} / 712 / 2 / 1049$

80. Kauffmann J, Pillai T, Shetty R, Myers PC, Goodman AA (2010) The Mass-Size Relation from Clouds to Cores. I. A New Probe of Structure in Molecular Clouds. ApJ, 712:1137-1146. https://doi.org/10.1088/0004$637 \mathrm{X} / 712 / 2 / 1137$

81. Pineda JE, Goodman AA, Arce HG, Caselli P, Foster JB, Myers PC, Rosolowsky EW (2010) Direct Observation of a Sharp Transition to Coherence in Dense Cores. ApJL, 712:L116-L121. https://doi.org/10.1088/20418205/712/1/L116

82. Schnee S, Enoch M, Noriega-Crespo A, Sayers J, Terebey S, Caselli P, Foster J, Goodman A, Kauffmann J, Padgett D, Rebull L, Sargent A, Shetty R (2010) The Dust Emissivity Spectral Index in the Starless Core TMC-1C. ApJ, 708:127-136. https://doi.org/10.1088/0004-637X/708/1/127

83. Li H-b., Dowell CD, Goodman A, Hildebrand R, Novak G (2009) Anchoring Magnetic Field in Turbulent Molecular Clouds. ApJ, 704:891-897. https://doi.org/10.1088/0004-637X/704/2/891

84. Pineda JE, Rosolowsky EW, Goodman AA (2009) The Perils of Clumpfind: The Mass Spectrum of Substructures in Molecular Clouds. ApJL, 699:L134-L138. https://doi.org/10.1088/0004-637X/699/2/L134

85. Shetty R, Kauffmann J, Schnee S, Goodman AA (2009) The Effect of Noise on the Dust Temperature-Spectral Index Correlation. ApJ, 696:676-680. https://doi.org/10.1088/0004-637X/696/1/676

86. Foster JB, Rosolowsky EW, Kauffmann J, Pineda JE, Borkin MA, Caselli P, Myers PC, Goodman AA (2009) Dense Cores in Perseus: The Influence of Stellar Content and Cluster Environment. ApJ, 696:298-319. https: //doi.org/10.1088/0004-637X/696/1/298

87. Shetty R, Kauffmann J, Schnee S, Goodman AA, Ercolano B (2009) The Effect of Line-of-Sight Temperature Variation and Noise on Dust Continuum Observations. ApJ, 696:2234-2251. https://doi.org/10.1088/0004$637 X / 696 / 2 / 2234$

88. Goodman AA, Pineda JE, Schnee SL (2009) The "True" Column Density Distribution in Star-Forming Molecular Clouds. ApJ, 692:91-103. https://doi.org/10.1088/0004-637X/692/1/91

89. Goodman AA, Rosolowsky EW, Borkin MA, Foster JB, Halle M, Kauffmann J, Pineda JE (2009) A role for self-gravity at multiple length scales in the process of star formation. Nature, 457:63-66. https://doi.org/10. 1038/nature07609

90. Schnee S, Li J, Goodman AA, Sargent AI (2008) Dust Emission from the Perseus Molecular Cloud. ApJ, 684:1228-1239. https://doi.org/10.1086/590375

91. Rosolowsky EW, Pineda JE, Kauffmann J, Goodman AA (2008) Structural Analysis of Molecular Clouds: Dendrograms. ApJ, 679:1338-1351. https://doi.org/10.1086/587685

92. Pineda JE, Caselli P, Goodman AA (2008) CO Isotopologues in the Perseus Molecular Cloud Complex: the X-factor and Regional Variations. ApJ, 679:481-496. https://doi.org/10.1086/586883 
93. Rosolowsky EW, Pineda JE, Foster JB, Borkin MA, Kauffmann J, Caselli P, Myers PC, Goodman AA (2008) An Ammonia Spectral Atlas of Dense Cores in Perseus. ApJS, 175:509-521. https://doi.org/10.1086/524299

94. Foster JB, Román-Zúñiga CG, Goodman AA, Lada EA, Alves J (2008) Hunting Galaxies to (and for) Extinction. ApJ, 674:831-845. https://doi.org/10.1086/524979

95. Schnee S, Caselli P, Goodman A, Arce HG, Ballesteros-Paredes J, Kuchibhotla K (2007) TMC-1C: An Accreting Starless Core. ApJ, 671:1839-1857. https://doi.org/10.1086/521577

96. Schnee S, Kauffmann J, Goodman A, Bertoldi F (2007) The Effect of Noise in Dust Emission Maps on the Derivation of Column Density, Temperature, and Emissivity Spectral Index. ApJ, 657:838-848. https://doi.org/ $10.1086 / 511054$

97. Goodwin SP, Kroupa P, Goodman A, Burkert A (2007) The Fragmentation of Cores and the Initial Binary Population. Protostars and Planets $V,: 133-147$.

98. Ridge NA, Di Francesco J, Kirk H, Li D, Goodman AA, Alves JF, Arce HG, Borkin MA, Caselli P, Foster JB, Heyer MH, Johnstone D, Kosslyn DA, Lombardi M, Pineda JE, Schnee SL, Tafalla M (2006) The COMPLETE Survey of Star-Forming Regions: Phase I Data. AJ, 131:2921-2933. https://doi.org/10.1086/503704

99. Ridge NA, Schnee SL, Goodman AA, Foster JB (2006) The COMPLETE Nature of the Warm Dust Shell in Perseus. ApJ, 643:932-944. https://doi.org/10.1086/502957

100. Schnee S, Bethell T, Goodman A (2006) Estimating the Column Density in Molecular Clouds with Far-Infrared and Submillimeter Emission Maps. ApJL, 640:L47-L50. https://doi.org/10.1086/503292

101. Foster JB, Goodman AA (2006) Cloudshine: New Light on Dark Clouds. ApJL, 636:L105-L108. https: //doi.org/10.1086/500131

102. Schnee SL, Ridge NA, Goodman AA, Li JG (2005) A COMPLETE Look at the Use of IRAS Emission Maps to Estimate Extinction and Dust Temperature. ApJ,634:442-450. https://doi.org/10.1086/491729

103. Schnee S, Goodman A (2005) Density and Temperature Structure of TMC-1C from 450 and 850 Micron Maps. ApJ, 624:254-266. https://doi.org/10.1086/429156

104. Goodman AA, Arce HG (2004) PV Cephei: Young Star Caught Speeding?. ApJ, 608:831-845. https: //doi.org/10.1086/383139

105. Padoan P, Goodman AA, Juvela M (2003) The Spectral Correlation Function of Molecular Clouds: A Statistical Test for Theoretical Models. ApJ, 588:881-893. https://doi.org/10.1086/374240

106. Arce HG, Goodman AA (2002) Bow Shocks, Wiggling Jets, and Wide-Angle Winds: A High-Resolution Study of the Entrainment Mechanism of the PV Cephei Molecular (CO) Outflow. ApJ, 575:928-949. https: //doi.org/10.1086/341426

107. Arce HG, Goodman AA (2002) The Great PV Cephei Outflow: A Case Study in Outflow-Cloud Interaction. ApJ, 575:911-927. https://doi.org/10.1086/341427

108. Ballesteros-Paredes J, Vázquez-Semadeni E, Goodman AA (2002) Velocity Structure of the Interstellar Medium as Seen by the Spectral Correlation Function. ApJ, 571:334-355. https://doi.org/10.1086/339875

109. Padoan P, Goodman A, Draine BT, Juvela M, Nordlund A, Rögnvaldsson ÖE (2001) Theoretical Models of Polarized Dust Emission from Protostellar Cores. ApJ, 559:1005-1018. https://doi.org/10.1086/322504

110. Padoan P, Kim S, Goodman A, Staveley-Smith L (2001) A New Method to Measure and Map the Gas Scale Height of Disk Galaxies. ApJL, 555:L33-L36. https://doi.org/10.1086/321735

111. Arce HG, Goodman AA (2001) The Episodic, Precessing Giant Molecular Outflow from IRAS 04239+2436 (HH 300). ApJ, 554:132-151. https://doi.org/10.1086/321334

112. Padoan P, Juvela M, Goodman AA, Nordlund $\AA(2001)$ The Turbulent Shock Origin of Proto-Stellar Cores. ApJ, 553:227-234. https://doi.org/10.1086/320636

113. Arce HG, Goodman AA (2001) The Mass-Velocity and Position-Velocity Relations in Episodic Outflows. ApJL, 551:L171-L174. https://doi.org/10.1086/320031 
114. Padoan P, Rosolowsky EW, Goodman AA (2001) The Effects of Noise and Sampling on the Spectral Correlation Function. ApJ, 547:862-871. https://doi.org/10.1086/318378

115. Weintraub DA, Goodman AA, Akeson RL (2000) Polarized Light from Star-Forming Regions. Protostars and Planets $I V,: 247-272$.

116. Rosolowsky EW, Goodman AA, Wilner DJ, Williams JP (1999) The Spectral Correlation Function: A New Tool for Analyzing Spectral Line Maps. ApJ, 524:887-894. https://doi.org/10.1086/307863

117. Arce HG, Goodman AA (1999) An Extinction Study of the Taurus Dark Cloud Complex. ApJ, 517:264-281. https://doi.org/10.1086/307168

118. Riess AG, Kirshner RP, Schmidt BP, Jha S, Challis P, Garnavich PM, Esin AA, Carpenter C, Grashius R, Schild RE, Berlind PL, Huchra JP, Prosser CF, Falco EE, Benson PJ, Briceño C, Brown WR, Caldwell N, dell'Antonio IP, Filippenko AV, Goodman AA, Grogin NA, Groner T, Hughes JP, Green PJ, Jansen RA, Kleyna JT, Luu JX, Macri LM, McLeod BA, McLeod KK, McNamara BR, McLean B, Milone AAE, Mohr JJ, Moraru D, Peng C, Peters J, Prestwich AH, Stanek KZ, Szentgyorgyi A, Zhao P (1999) BVRI Light Curves for 22 Type IA Supernovae. AJ, 117:707-724. https://doi.org/10.1086/300738

119. Arce HG, Goodman AA (1999) Measuring Galactic Extinction: A Test. ApJL, 512:L135-L138. https: //doi.org/10.1086/311885

120. Barranco JA, Goodman AA (1998) Coherent Dense Cores. I. $\mathrm{NH}_{3}$ Observations. ApJ, 504:207-222. https: //doi.org/10.1086/306044

121. Goodman AA, Barranco JA, Wilner DJ, Heyer MH (1998) Coherence in Dense Cores. II. The Transition to Coherence. ApJ, 504:223-246. https://doi.org/10.1086/306045

122. Arce HG, Goodman AA, Bastien P, Manset N, Sumner M (1998) The Polarizing Power of the Interstellar Medium in Taurus. ApJL, 499:L93-L97. https://doi.org/10.1086/311337

123. Goodman AA, Barranco JA, Wilner DJ, Heyer MH (1998) Velocity Coherence in Dense Cores. Astrophysical Letters and Communications, 37:109.

124. Lazarian A, Goodman AA, Myers PC (1997) On the Efficiency of Grain Alignment in Dark Clouds. ApJ, 490:273-280.

125. Pound MW, Goodman AA (1997) Kinematics of the Ursa Major Molecular Clouds. ApJ, 482:334-354.

126. Troland TH, Crutcher RM, Goodman AA, Heiles C, Kazes I, Myers PC (1996) The Magnetic Fields in the Ophiuchus and Taurus Molecular Clouds. ApJ, 471:302. https://doi.org/10.1086/177970

127. Goodman AA, Whittet DCB (1995) A Point in Favor of the Superparamagnetic Grain Hypothesis. ApJL, 455:L181. https://doi.org/10.1086/309840

128. Goodman AA, Jones TJ, Lada EA, Myers PC (1995) Does Near-Infrared Polarimetry Reveal the Magnetic Field in Cold Dark Clouds?. ApJ, 448:748. https://doi.org/10.1086/176003

129. Myers PC, Goodman AA, Gusten R, Heiles C (1995) Observations of magnetic fields in diffuse clouds. ApJ, 442:177-185. https://doi.org/10.1086/175433

130. Goodman AA (1995) Mapping magnetic fields in the ISM: infrared and sub-mm polarimetry.. The Physics and Chemistry of Interstellar Molecular Clouds, 459:82-85. https://doi.org/10.1007/BFb0102096

131. Ladd EF, Myers PC, Goodman AA (1994) Dense cores in dark clouds. 10: Ammonia emission in the Perseus molecular cloud complex. ApJ, 433:117-130. https://doi.org/10.1086/174629

132. Goodman AA, Heiles C (1994) The magnetic field in the Ophiuchus dark cloud complex. ApJ, 424:208-221. https://doi.org/10.1086/173884

133. Goodman AA, Benson PJ, Fuller GA, Myers PC (1993) Dense cores in dark clouds. VIII - Velocity gradients. ApJ, 406:528-547. https://doi.org/10.1086/172465

134. Crutcher RM, Troland TH, Goodman AA, Heiles C, Kazes I, Myers PC (1993) OH Zeeman observations of dark clouds. ApJ, 407:175-184. https://doi.org/10.1086/172503 
135. Goodman AA, Jones TJ, Lada EA, Myers PC (1992) The structure of magnetic fields in dark clouds - Infrared polarimetry in B216-217. ApJ, 399:108-113. https://doi.org/10.1086/171907

136. Myers PC, Fuller GA, Goodman AA, Benson PJ (1991) Dense cores in dark clouds. VI - Shapes. ApJ, 376:561-572. https://doi.org/10.1086/170305

137. Myers PC, Goodman AA (1991) On the dispersion in direction of interstellar polarization. ApJ, 373:509-524. https://doi.org/10.1086/170070

138. Goodman A (1990) Thermal shape fluctuations at constant energy. Nuclear Physics A, 520:567. https: //doi.org/10.1016/0375-9474(90)91175-Q

139. Goodman AA, Bastien P, Menard F, Myers PC (1990) Optical polarization maps of star-forming regions in Perseus, Taurus, and Ophiuchus. ApJ, 359:363-377. https://doi.org/10.1086/169070

140. Bastien P, Goodman AA, Myers PC, Ménard F (1989) Linear polarization maps of three molecular clouds. JRASC, 83:298.

141. Goodman AA, Crutcher RM, Heiles C, Myers PC, Troland TH (1989) Measurement of magnetic field strength in the dark cloud Barnard 1. ApJL, 338:L61-L64. https://doi.org/10.1086/185401

142. Goodman AA, Myers PC, Crutcher RM, Heiles C, Kazes I (1989) Measurement of the magnetic field in the molecular cloud B1. The Physics and Chemistry of Interstellar Molecular Clouds - mm and Sub-mm Observations in Astrophysics, 331:182-185. https://doi.org/10.1007/BFb0119475

143. Myers PC, Goodman AA (1988) Magnetic molecular clouds - Indirect evidence for magnetic support and ambipolar diffusion. ApJ, 329:392-405. https://doi.org/10.1086/166385

144. Myers PC, Goodman AA (1988) Evidence for magnetic and virial equilibrium in molecular clouds. ApJL, 326:L27-L30. https://doi.org/10.1086/185116

145. Goodman AA, Myers PC (1988) Magnetic and Virial Equilibrium in Molecular Clouds. Molecular Clouds, Milky-Way and External Galaxies, 315:128. https://doi.org/10.1007/3-540-50438-9_250

146. Robitaille T, Beaumont C, Qian P, Borkin M, Goodman A (2017) glueviz v0.13.1: multidimensional data exploration. https://doi.org/10.5281/zenodo.1237692

147. Chen HH-H, Pineda JE, Offner SSR, Goodman AA, Burkert A, Friesen RK, Rosolowsky E, Scibelli S, Shirley Y (2019) Droplets II: Internal Velocity Structures and Potential Rotational Motions in Coherent Cores. arXiv e-prints, :arXiv:1908.04367.

148. Fissel LM, Ade PAR, Angilè FE, Ashton P, Benton SJ, Chen C-Y, Cunningham M, Devlin MJ, Dober B, Friesen R, Fukui Y, Galitzki N, Gandilo NN, Goodman A, Green C-E, Jones P, Klein J, King P, Korotkov AL, Li Z-Y, Lowe V, Martin PG, Matthews TG, Moncelsi L, Nakamura F, Netterfield CB, Newmark A, Novak G, Pascale E, Poidevin F, Santos FP, Savini G, Scott D, Shariff JA, Soler JD, Thomas NE, Tucker CE, Tucker GS, Ward-Thompson D, Zucker C (2019) Relative Alignment between the Magnetic Field and Molecular Gas Structure in the Vela C Giant Molecular Cloud Using Low- and High-density Tracers. ApJ, 878(2):110. https://doi.org/10.3847/1538-4357/ab1eb0

149. Harju J, Pineda JE, Vasyunin AI, Caselli P, Offner SSR, Goodman AA, Juvela M, Sipilae O, Faure A, Le Gal R, Hily-Blant P, Alves J, Bizzocchi L, Burkert A, Chen H, Friesen RK, Guesten R, Myers PC, Punanova A, Rist C, Rosolowsky E, Schlemmer S, Shirley Y, Spezzano S, Vastel C, Wiesenfield L (2019) Efficient methanol desorption in shear instability. arXiv e-prints, :arXiv:1903.11298. 